\title{
Mandatory nonmedical switching from originator to biosimilar infliximab in patients with inflammatory arthritis and psoriasis in British Columbia: a cohort study
}

\author{
Anat Fisher MD PhD, Jason D. Kim MPH, Colin R. Dormuth ScD
}

Abstract

Background: In 2019, British Columbia's public drug plan, PharmaCare, was the first in Canada to implement a nonmedical switching policy from originator infliximab to its biosimilar, for patients with inflammatory arthritis or psoriasis. We aimed to detect signals of impact on health services utilization during the first year of policy implementation and to provide early data to policy-makers.

Methods: We constructed cohorts of users of originator infliximab: 3 historical cohorts (2016-2018) and 1 policy cohort (2019). We extracted data from BC Ministry of Health databases from 2015 to 2020, as we followed each cohort for 365 days from May 27 of each cohort's respective year. We excluded patients with gastrointestinal conditions and those not covered by PharmaCare. We examined the cumulative incidence of infliximab prescription refills, switching to other biologic drugs and use of additional health services. A log-likelihood ratio of 1.96 compared with the null hypothesis was used as the threshold for differences between the policy cohort and the historical cohorts.

Results: The study included a total of 572 unique patients: 520 in the 2016 historical cohort, 461 in the 2017 historical cohort, 423 in the 2018 historical cohort and 377 in the policy cohort (with some patients included in multiple cohorts; 335 [58.6\%] were included in all 4 cohorts). During months 8 and 9 of follow-up, a transient signal was observed in infliximab refills $(7.2 \%$ decrease in refilling infliximab for the fourth time for the policy cohort, log-likelihood ratio > 1.96). An anticipated increase in visits to specialists was observed from month 4 forward (15.0\%, log-likelihood ratio > 1.96). No signal was observed for increased use of other health services (loglikelihood ratio < 1.96).

Interpretation: Early monitoring did not detect signals of negative impacts on health services use during the first year of the policy. Detailed, longer-term cohort studies and hypothesis-testing methods could provide additional assurance about the safety of the policy.

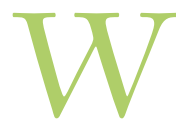
orldwide spending on biologic medications is high and increasing, ${ }^{1-3}$ and governments face the challenge of providing these medications and other treatments within limited budgets. The use of biosimilar medications has the potential to reduce $\operatorname{costs}^{4-6}$ while providing clinical benefits similar to those of the originator medications. ${ }^{7-9}$ To encourage switching from originator biologics to biosimilar medications, some governments are implementing voluntary ${ }^{10}$ or mandatory ${ }^{11-14}$ nonmedical switching policies. Despite evidence that switching is not associated with negative health impacts, ${ }^{15,16}$ many patients and physicians are concerned that switching will reduce the effectiveness of treatment or cause adverse effects (or both). ${ }^{17,18}$ Unintended negative effects may be a particular concern when switching is mandatory, regardless of a patient's clinical presentation or medical history. ${ }^{19,20}$ Close monitoring of the impacts of mandatory switching policies is needed to provide early data on the safety of these policies.

On May 27, 2019, British Columbia was the first Canadian province to implement a mandatory nonmedical switch policy from an originator to a biosimilar medication. ${ }^{21}$ Phase 1 of the BC Biosimilars Initiative targeted patients with rheumatoid arthritis, ankylosing spondylitis, psoriatic arthritis or plaque psoriasis who were treated with the originator infliximab and reimbursed by the provincial drug plan, PharmaCare. In BC, the costs for infliximab are covered under a PharmaCare Special Authority process. ${ }^{22}$ Since May 27, 2019, only the biosimilar infliximab has been approved in response to new or renewal Special Authority requests for patients with inflammatory arthritis or psoriasis. Patients with existing approval were required to switch to the biosimilar infliximab during a 6-month transition period, and after Nov. 25, 2019, PharmaCare no longer covered the costs of originator infliximab.

\section{Competing interests: None declared.}

This article has been peer reviewed.

Correspondence to: Anat Fisher, anat.fisher@ubc.ca

CMAJ Open 2022 February 15. DOI:10.9778/cmajo.20200319 
The launch of the Biosimilars Initiative was followed by real-time monitoring of its impacts. ${ }^{23-25}$ Some impacts were expected, such as an increase in visits to specialists during the 6-month transition period or immediately thereafter because of patient-physician consultations needed to monitor a switch to a biosimilar. ${ }^{10,14}$ Also, a shift in health services utilization was expected beginning Mar. 17, 2020, when the provincial government announced a state of emergency due to the COVID-19 pandemic. ${ }^{26}$ Specifically, we anticipated a decrease in visits to physicians and hospitals, ${ }^{27}$ as well as a change in the refilling of prescriptions due to stockpiling (panic buying) ${ }^{28,29}$ or manufacturing disruption. ${ }^{30}$

Our objective was to monitor signals of intended and unintended impacts of the Biosimilars Initiative on drug and health services utilization during the first year of implementation.

\section{Methods}

\section{Setting and study design}

The province of $\mathrm{BC}$ has a publicly funded health system that provides medically necessary health care services. All residents, regardless of age and income, are eligible to register with the provincial drug plan, PharmaCare, which covers eligible prescription drugs, certain medical supplies and pharmacy services. The largest plan, Fair PharmaCare, is income-based, and patients and families are required to spend their deductible level each year on eligible costs before Fair PharmaCare starts to help with these costs.

We designed a rapid monitoring project to detect signals of the impacts of the 2019 infliximab coverage policy in BC on health services utilization, but not to accept or reject a hypothesis of harm in the presence of sampling error. We monitored routinely collected administrative data to ensure that changes in health services utilization were promptly recognized and investigated. Given the large amount of data being collected and analyzed, we developed detection algorithms to facilitate our analyses. The signals detected by these algorithms did not necessarily imply negative impacts of the policy or switching to the biosimilar infliximab; rather, they allowed quick and effective screening of utilization data for outliers.

\section{Participants}

We created 4 fixed cohorts of users of the originator infliximab: a policy cohort, which was exposed to the Biosimilars Initiative, and 3 historical control cohorts, which represented historical patterns of health care utilization before the policy shift. We included users who filled at least 1 prescription for the originator infliximab using a look-back period of 6 months from May 27, 2019, for the policy cohort and from the same date in 2016, 2017 and 2018, respectively, for the historical cohorts. We excluded patients not targeted by the policy (i.e., those who had a gastrointestinal condition [Crohn disease or ulcerative colitis]). The remaining patients were likely treated for rheumatoid arthritis, ankylosing spondylitis, psoriatic arthritis or psoriasis. We also excluded patients who, during the look-back period, discontinued use of originator infliximab, switched away from use of this drug or did not have PharmaCare coverage for originator infliximab.
Additional details on the cohorts are provided in Appendix 1, Table S1 (available at www.cmajopen.ca/content/10/1/E109/ suppl/DC1). Patients were eligible for inclusion in more than 1 cohort. We followed patients in each cohort for 365 days.

\section{Data sources}

This rapid monitoring study was a population-based cohort study using linkable and anonymized administrative data (Appendix 1, Table S2). Databases we used included the BC Ministry of Health's Pharmanet (prescriptions filled at community pharmacies) and the Medical Services Plan databases (registration [demographic characteristics and patient enrolment in the provincial Medical Services Plan] and payment information and premium billing [visits to physicians]) and the Canadian Institute for Health Information's Discharge Abstract Database (hospital admissions) and National Ambulatory Care Reporting System database (visits to emergency departments).

The protocol was published previously. ${ }^{24}$ Deviations from the protocol are listed in Appendix 1, Table S3.

\section{Measures of health services utilization}

We measured the daily cumulative incidence of multiple outcomes from May 27 (day 1) and during the following 364 days. We measured the daily outcome separately for each of the 4 cohorts. We present cumulative incidence as the percentage of patients in each cohort who experienced the outcome by a specific day of follow-up (1-365).

Drug utilization outcomes were refilling a prescription for infliximab and switching to a different biologic or targeted synthetic disease-modifying antirheumatic drug (DMARD) (Appendix 1, Table S4).

Health services utilization outcomes were visits to physicians in an outpatient setting, visits to rheumatologists or dermatologists (outpatient setting), first visit to an emergency department after cohort entry and first discharge from hospital. For the policy cohort, hospital data were not available for the last 2 months of the post-policy intervention period.

We present the secondary outcomes as average cumulative quantity per patient. We calculated these values as the sum of quantities (such as prescription refilled or milligrams) that were recorded by a specific follow-up day (1-365) divided by the number of patients in each cohort. Secondary outcomes were the average cumulative dose of infliximab and the average cumulative number of prescriptions filled for antiinflammatory medications: conventional synthetic DMARDs, nonsteroidal anti-inflammatory drugs (NSAIDs) or oral corticosteroids. The full list of medications is presented in Appendix 1, Table S4.

\section{Statistical analysis}

To study health services utilization in patients affected by the Biosimilars Initiative, we compared services on a daily basis; for example, a service measured on May 28, 2019 (policy cohort), was compared with the average measure from May 28 of the years 2016, 2017 and 2018 (historical cohorts). This assessment controlled for seasonal effects, such as the effect of annual PharmaCare deductibles that led to increased medication 
stockpiling toward the end of a calendar year. ${ }^{31}$ Averaged cumulative incidence patterns of outcomes from the historical cohorts represented the expected patterns for the policy cohort in the absence of a policy impact. We calculated the difference in daily cumulative incidence between the policy cohort and the average of the 3 historical cohorts.

We identified potential signals for policy-induced differences between the policy and control cohorts using a loglikelihood ratio. We used likelihood ratios instead of test statistics because interpretation of the former remains the same regardless of why or how many times a data analysis was updated (with our analyses being updated daily). ${ }^{32}$ As per our published protocol, ${ }^{24}$ a cumulative incidence difference between the policy and the average of the historical cohorts was considered a signal for a potential policy-induced difference if the likelihood of the observed cumulative incidence difference was at least $\mathrm{e}^{(\mathrm{z}=1.96)}$ or 7.1 times greater than the likelihood of a cumulative incidence difference of 0 , given the data. ${ }^{33} \mathrm{We}$ presented the data to policy-makers on a monthly basis; therefore, log-likelihood ratios sustained above the 1.96 threshold for less than a month $(31 \mathrm{~d})$ were not considered to represent a signal. Additional details on computation of the $\log$-likelihood ratios are provided in Appendix 1, Table S5.

\section{Ethics approval}

The study protocol ${ }^{24}$ was approved by the University of British Columbia Clinical Research Ethics Board (UBC CREB number H19-02377).
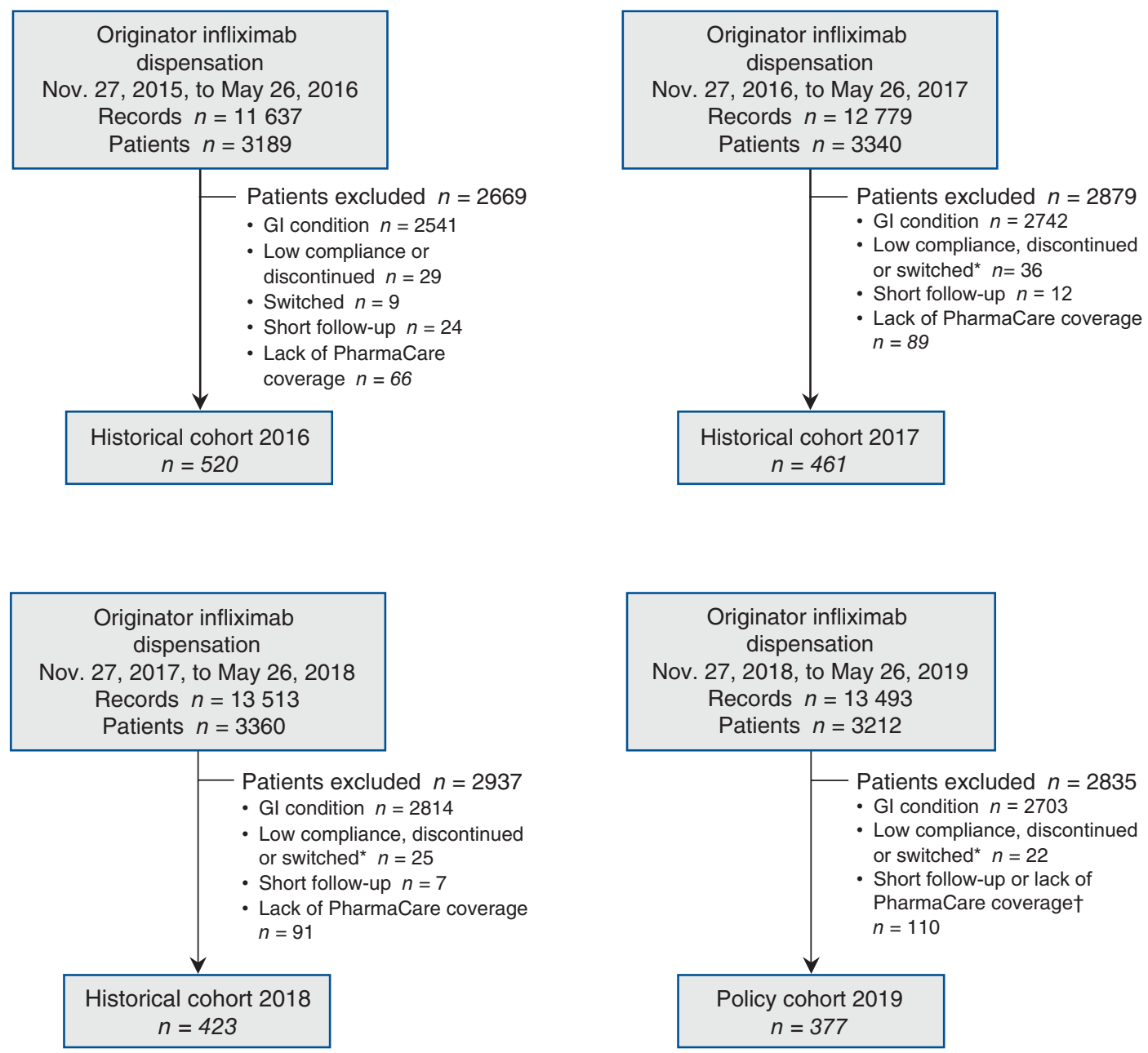

Figure 1: Flow chart of cohort creation. The cohorts consist of users of the originator infliximab. Gastrointestinal (GI) conditions included ulcerative colitis and Crohn disease. Low compliance or infliximab discontinuation was defined as no medication supply on May 27 combined with no refill in the 84 days before (excluding) May 27. Switching was defined as a refill of a different biologic anti-inflammatory medication or the biosimilar version of infliximab. Short follow-up was defined as less than 1 month of medical plan enrolment starting on the cohort entry date (May 27). PharmaCare coverage was determined based on all originator infliximab refills during the 6-month period before May 27. ${ }^{*}$ The number of patients who switched was less than 6 . †The number of patients with follow-up duration shorter than 1 month was less than 6. 


\section{Results}

The source population consisted of 5431788 individuals who had been enrolled in BC's Medical Services Plan between November 2015 and May 2019. Patients with Crohn disease or ulcerative colitis accounted for $82.4 \%$ of the patients treated with the originator infliximab; these patients were not targeted by phase 1 of the Biosimilars Initiative and were excluded from the analysis. The number of patients was 520 in the 2016 historical cohort, 461 in the 2017 historical cohort, 423 in the 2018 historical cohort and 377 in the policy cohort (Figure 1). Overall, 335 (58.6\%) of the 572 unique patients in the study were included in all 4 cohorts.

The median age in the 4 cohorts ranged between 57.5 and 60.0 years, and the majority of patients were female $(53.1 \%$ to
$55.6 \%$ ) (Table 1). Rheumatoid arthritis was the most common diagnosis $(50.4 \%$ to $53.5 \%$ of patients in the various cohorts), and psoriasis the least common (3.3\% to $4.0 \%)$. Compared with the average of the 3 historical cohorts, patients in the policy cohort used fewer conventional synthetic anti-inflammatory medications (Table 1). No other important differences in baseline characteristics were observed between the cohorts.

By the end of the 6-month transition period, 233 (61.8\%) of the 377 patients in the policy cohort had switched to biosimilar infliximab, and by the end of the 1-year follow-up, 329 $(87.3 \%)$ had switched (Figure 2$)$.

\section{Infliximab and health services utilization}

Most patients refilled their infliximab prescriptions at least 4 times by the end of the follow-up period (Figure 3A-D).

\section{Table 1: Cohorts of users of the originator infliximab}

\begin{tabular}{|c|c|c|c|c|}
\hline \multirow[b]{2}{*}{ Characteristic } & \multicolumn{4}{|c|}{ Infliximab cohort; no. (\%) of patients* } \\
\hline & $\begin{array}{c}\text { Historical cohort } \\
2016 \\
n=520\end{array}$ & $\begin{array}{c}\text { Historical cohort } \\
\begin{array}{c}2017 \\
n=461\end{array}\end{array}$ & $\begin{array}{c}\text { Historical cohort } \\
2018 \\
n=423\end{array}$ & $\begin{array}{c}\text { Policy cohort } \\
2019 \\
n=377\end{array}$ \\
\hline \multicolumn{5}{|l|}{ Age, yr } \\
\hline Mean \pm SD & $56.4 \pm 15.7$ & $57.4 \pm 16.0$ & $58.3 \pm 15.6$ & $58.6 \pm 15.7$ \\
\hline Median (range) & $57.5(6.0-91.0)$ & $58.0(4.0-90.0)$ & $59.0(7.0-91.0)$ & $60.0(8.0-91.0)$ \\
\hline \multicolumn{5}{|l|}{ Sex } \\
\hline Female & $289(55.6)$ & $247(53.6)$ & $228(53.9)$ & $200(53.1)$ \\
\hline Male & $231(44.4)$ & $214(46.4)$ & $195(46.1)$ & $177(46.9)$ \\
\hline \multicolumn{5}{|l|}{ Most likely diagnosis } \\
\hline Any rheumatologic diagnosis & $480(92.3)$ & $422(91.5)$ & $387(91.5)$ & $345(91.5)$ \\
\hline Rheumatoid arthritis & $278(53.5)$ & $242(52.5)$ & $218(51.5)$ & $190(50.4)$ \\
\hline Ankylosing spondylosis & $115(22.1)$ & $109(23.6)$ & $101(23.9)$ & $90(23.9)$ \\
\hline Psoriatic arthritis & $45(8.7)$ & $41(8.9)$ & $38(9.0)$ & $30(8.0)$ \\
\hline Psoriasis & $19(3.7)$ & $15(3.3)$ & $17(4.0)$ & $15(4.0)$ \\
\hline Undetermined & $21(4.0)$ & $24(5.2)$ & $19(4.5)$ & $17(4.5)$ \\
\hline Time from first infliximab use, yr, mean \pm SD & $7.2 \pm 4.5$ & $8.5 \pm 4.4$ & $9.6 \pm 4.3$ & $10.6 \pm 4.3$ \\
\hline \multicolumn{5}{|l|}{ Health services utilization in previous year, mean \pm SD } \\
\hline \multicolumn{5}{|l|}{ No. of different medications } \\
\hline Any medication $†$ & $9.3 \pm 6.0$ & $9.3 \pm 6.2$ & $8.7 \pm 5.4$ & $8.7 \pm 5.6$ \\
\hline Biologic anti-inflammatory medications $\ddagger$ & $1.1 \pm 0.3$ & $1.0 \pm 0.1$ & $1.0 \pm 0.0$ & $1.0 \pm 0.0$ \\
\hline Conventional synthetic anti-inflammatory medications§ & $0.8 \pm 0.8$ & $0.8 \pm 0.8$ & $0.8 \pm 0.8$ & $0.7 \pm 0.7$ \\
\hline No. of visits to physicians? & $23.2 \pm 16.7$ & $23.1 \pm 17.9$ & $21.7 \pm 14.6$ & $20.9 \pm 15.0$ \\
\hline No. of nights in hospital ${ }^{* *}$ & $1.0 \pm 5.2$ & $0.8 \pm 3.9$ & $0.6 \pm 3.6$ & $0.5 \pm 2.6$ \\
\hline \multicolumn{5}{|c|}{$\begin{array}{l}\text { SD = standard deviation. } \\
\text { *Except where indicated otherwise. } \\
\text { †Based on non-brand names of prescription medications. } \\
\text { †Biologic anti-inflammatory medications were adalimumab, etanercept, certolizumab, golimumab, abatacept, tocilizumab, anakinra, tofacitinib, rituximab, ustekinumab, } \\
\text { secukinumab, ixekizumab, brodalumab and guselkumab. } \\
\text { §Conventional synthetic anti-inflammatory medications were methotrexate, hydroxychloroquine, leflunomide, sulfasalazine, minocycline, azathioprine, auranofin, } \\
\text { chloroquine, cyclophosphamide, cyclosporine, gold sodium thiomalate, mycophenolate and penicillamine. } \\
\text { IVisits to physician of any specialty, in an outpatient setting, are included, regardless of whether the subsequent visit was to the same physician. } \\
\text { **Includes all nights in hospital, regardless of whether the admission was associated with an emergency department visit. }\end{array}$} \\
\hline
\end{tabular}




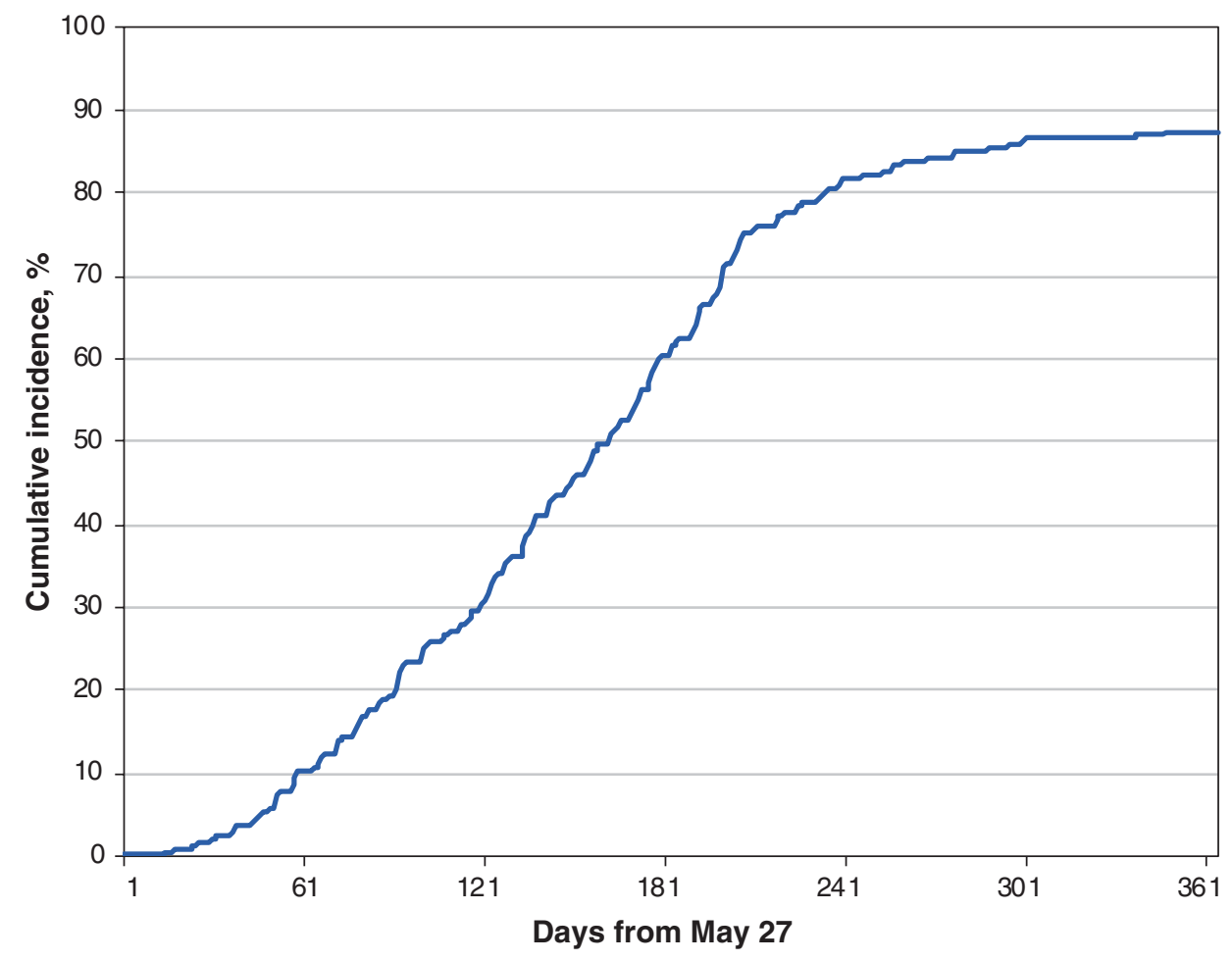

Figure 2: Cumulative incidence of switching to the biosimilar infliximab, policy cohort $(n=377)$.

The cumulative incidences for the first 3 infliximab refills were similar in the policy and historical cohorts (log-likelihood ratios $<1.96)$. We observed a transient and small signal for refilling infliximab for the fourth time during follow-up: a decline of $7.2 \%$ in patients from the policy cohort during days 223 to 275 (months 8 and 9, Jan. 14 to Feb. 25) (Figure 3D and Appendix 1, Figure S1). In the following days, the incidence of refilling infliximab for the fourth time increased, and the loglikelihood ratios decreased below the 1.96 threshold for the remaining follow-up duration. This pattern was not associated with a significant change in the average cumulative dose of infliximab (Appendix 1, Figure S2). In addition, we observed no signal for difference between the policy cohort and the average of the historical cohorts in relation to switching to a different biologic or targeted synthetic DMARD (Figure 3E).

Trends in visits to any physician in an outpatient setting for the policy cohort were similar to the historical trends, and nearly all patients had 4 visits (Figures 4A-D). Approximately $90 \%$ of patients from the historical cohorts had a first visit to a specialist (Figure $4 \mathrm{E}$ ) and $67 \%$ to $74 \%$ had a second visit to a specialist (Figure 4F). Patients in the policy cohort made a first visit to a specialist earlier than patients in the historical cohorts, with a signal detected from month 4 onward (Figure $4 \mathrm{E}$ and Appendix 1, Figure S3). By the end of the 1-year follow-up period, the differences diminished (and the loglikelihood ratio fell below the threshold of 1.96). In addition, more patients in the policy cohort visited a specialist twice compared with patients in the historical cohorts (up to an absolute increase of $+15 \%$ ), and the log-likelihood ratios remained above the 1.96 threshold from day 155 (month 6) onward (Figure 4F and Appendix 1, Figure S3). Among patients with 2 visits to a specialist during the follow-up periods, the first 2 visits were to the same specialist for $82 \%$ of patients in the historical cohorts from 2016 and 2017, for $88 \%$ of those in the historical cohort from 2018 and for $91 \%$ of patients in the policy cohort.

Patients' first visits to an emergency department and first discharges from hospital in the policy cohort were similar to the average of the historical cohorts (Figure 5, log-likelihood ratios $<1.96)$. The cumulative incidences of the first visit to an emergency department ranged between 21\% (historical cohorts for 2016 and 2018) and 23\% (historical cohort for 2017 ) by the end of the 1 -year follow-up period. The cumulative incidences of the first discharge from hospital ranged between $7.8 \%$ (historical cohort for 2017) and 10\% (historical cohort for 2016) by the end of 10 months of follow-up.

\section{Other medication utilization}

Compared with the historical cohorts, we found no increase in the use of other anti-inflammatory medications in the policy cohort. The policy cohort received fewer refills of conventional synthetic DMARDs and NSAIDs compared with the average of the historical cohorts, but all cohorts had similar patterns of oral corticosteroid use (Appendix 1, Figure S4). 
A

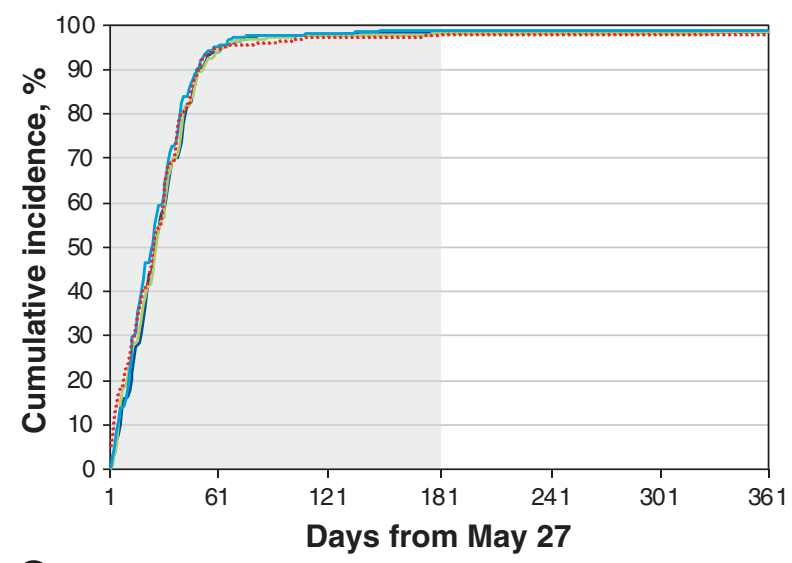

C

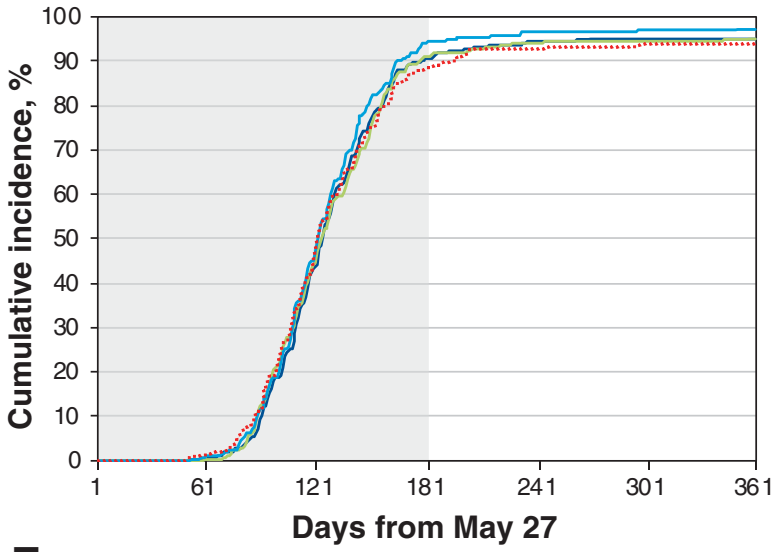

$\mathbf{E}$

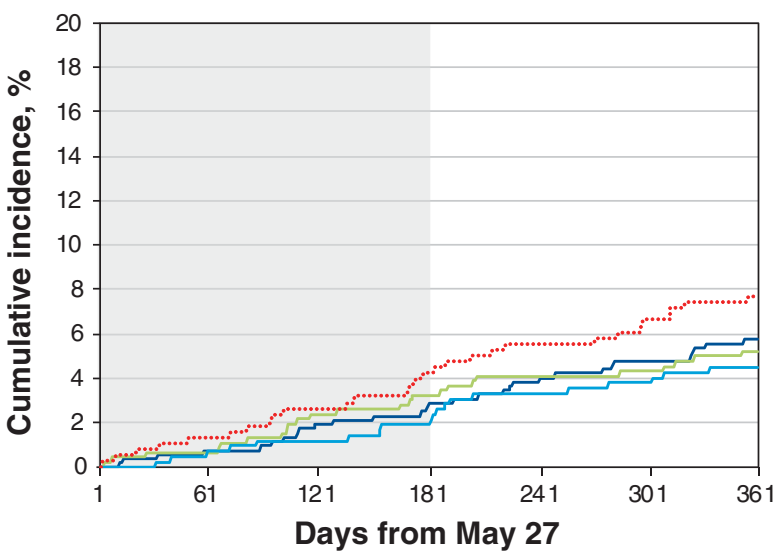

B

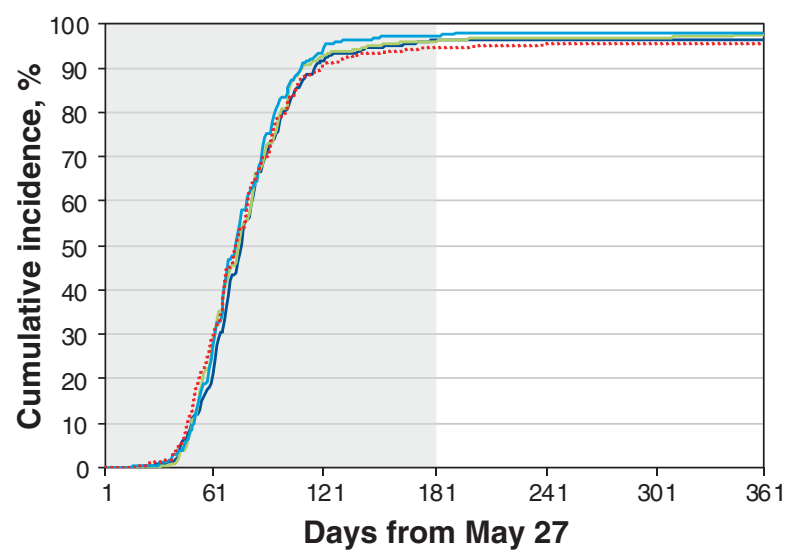

D

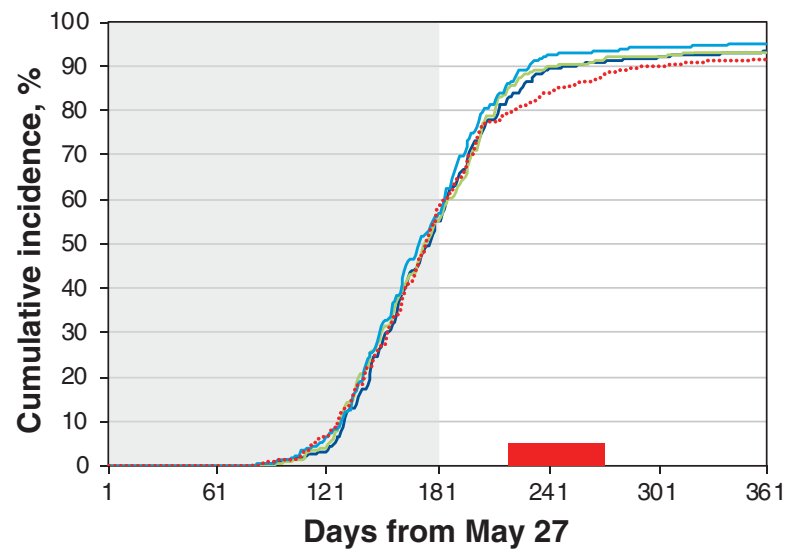

Transition period $\square$ Log-likelihood ratio $>1.96$ (signal)

- Historical cohort 2016, $n=520$ - Historical cohort 2017, $n=461$

- Historical cohort 2018, $n=423 \quad \cdots$ Policy cohort 2019, $n=377$

Figure 3: Cumulative incidence of filling the first $(A)$, second $(B)$, third $(C)$ and fourth $(D)$ prescriptions of infliximab and switching to a different biologic or targeted synthetic disease-modifying antirheumatic drug $(E)$ during the 1-year follow-up period, by cohort. Cumulative incidence is expressed as the percentage of patients in each cohort who experienced the outcome by day of follow-up. The 6-month transition period of the policy is shaded in grey. Days with log-likelihood ratios comparing the policy cohort and the average of the historical cohorts above the threshold of 1.96 are marked by a red block. 
A

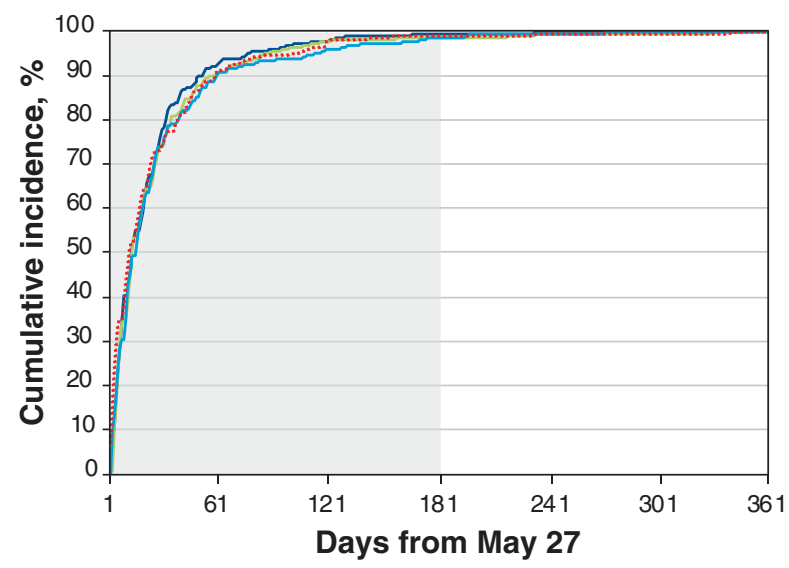

C

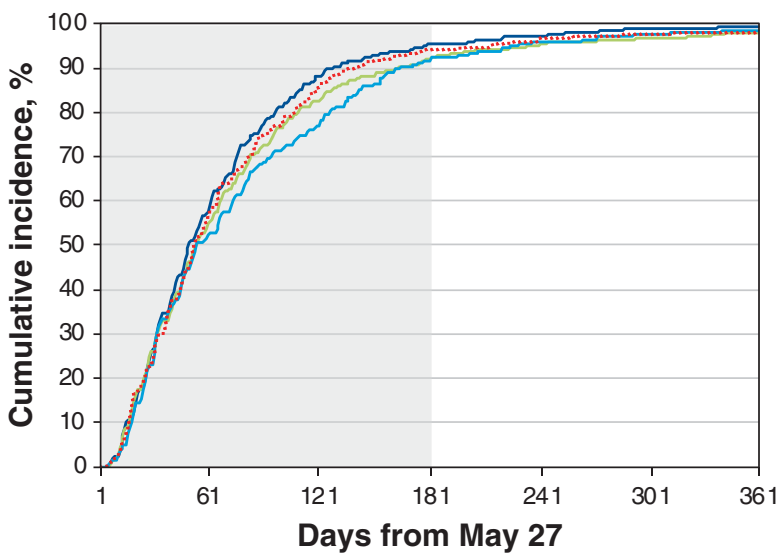

E

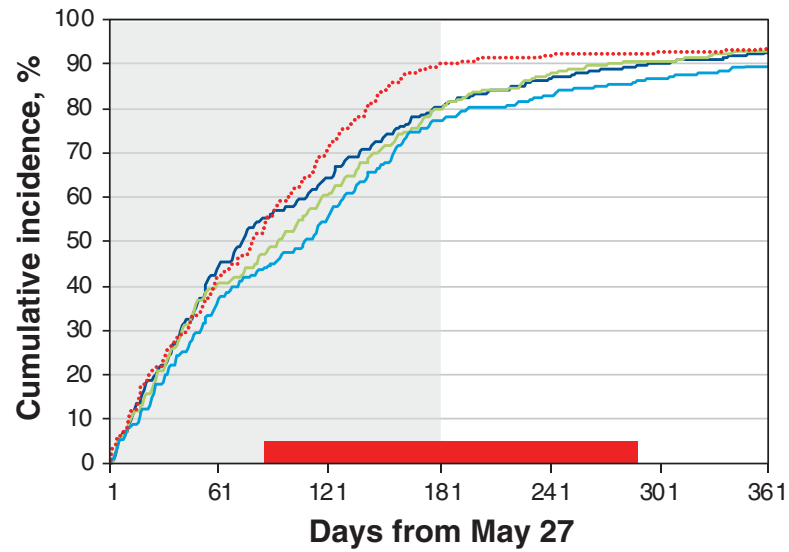

B

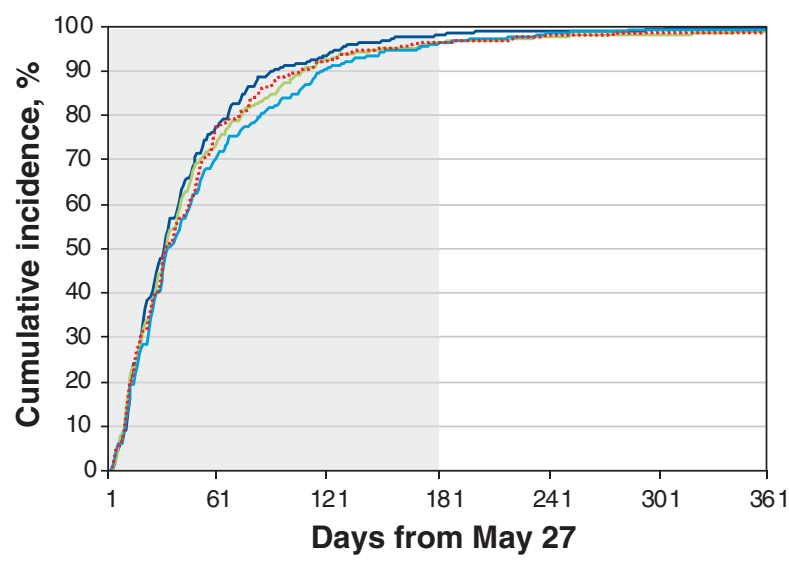

D

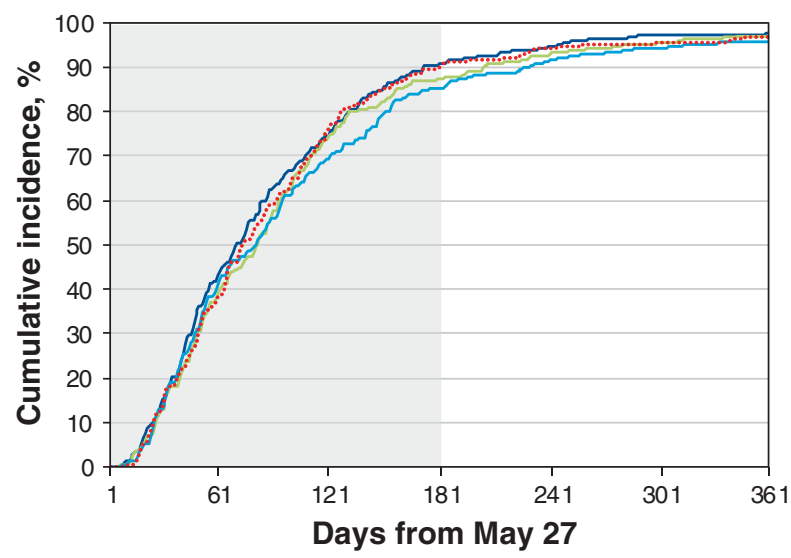

$\mathbf{F}$

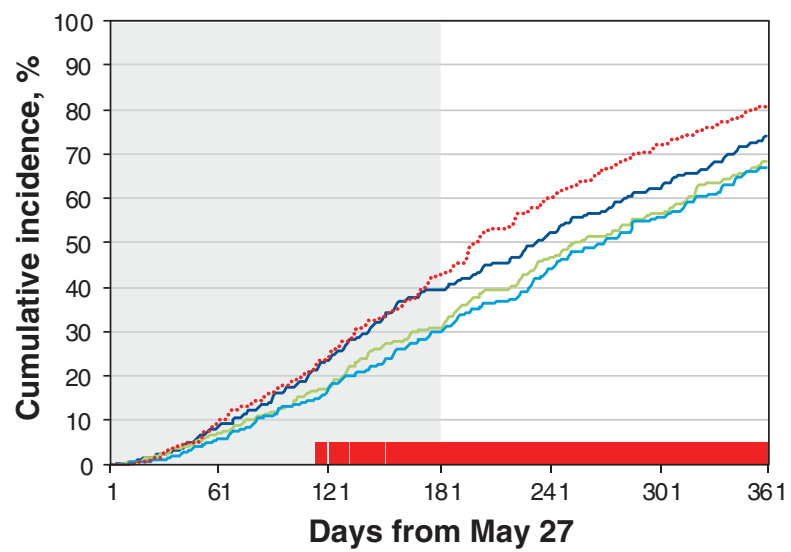

Figure 4: Cumulative incidence of the first $(A)$, second $(B)$, third $(C)$ and fourth $(D)$ visits to a physician and the first $(E)$ and second $(F)$ visits to a specialist during the 1-year follow-up period, by cohort. Cumulative incidence is expressed as the percentage of patients in each cohort who experienced the outcome by day of follow-up. The 6-month transition period of the policy is shaded in grey. Days with log-likelihood ratios comparing the policy cohort and the average of the historical cohorts above the threshold of 1.96 are marked by a red block. 


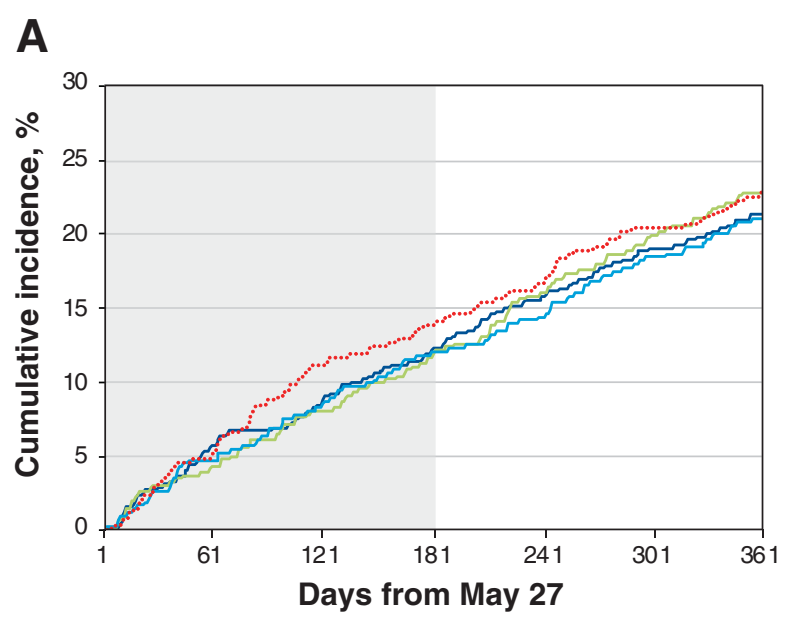

B

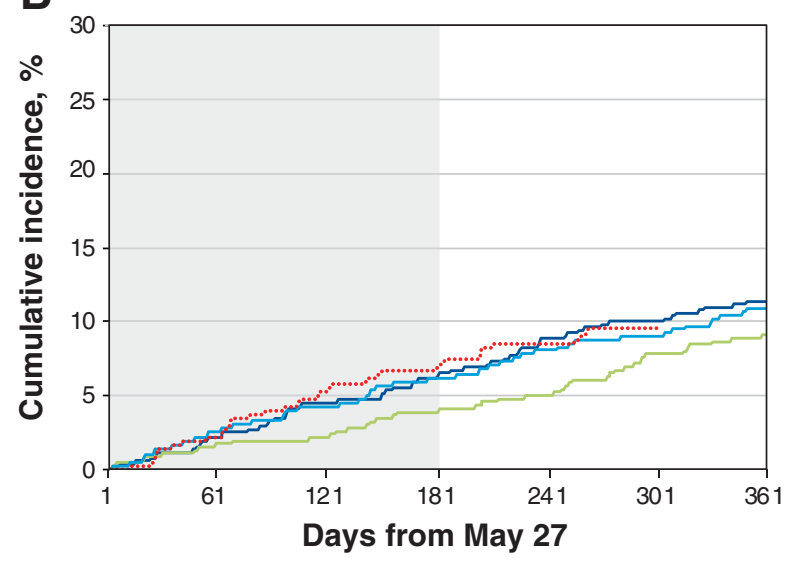

Figure 5: Cumulative incidence of the first visit to an emergency department $(A)$ and the first discharge from hospital (B) during the 1-year follow-up period, by cohort. Cumulative incidence is expressed as the percentage of patients in each cohort who experienced the outcome by day of follow-up. The 6-month transition period of the policy is shaded in grey. Hospital data were available until Mar. 26, 2020.

Similar patterns in the use of conventional synthetic DMARDs and NSAIDs were also observed in a subgroup analysis of patients with rheumatoid arthritis (Appendix 1, Figure S5).

\section{Interpretation}

This monitoring of infliximab users targeted by phase 1 of the BC Biosimilars Initiative provided early data during the first year after the policy launch. We observed no signal for a negative impact of the infliximab policy on health services utilization during the first year of follow-up; more specifically, we found no increase in switching to a different biologic or targeted synthetic
DMARD, no increase in the use of other anti-inflammatory medications and no increases in visits to emergency departments or in hospital admissions. We did, however, detect a transient decrease in refills of infliximab and an expected increase in visits to specialists. The COVID-19 pandemic had no detectable impact on health services utilization.

Seasonal trends do not explain the signal of a small transient decrease in the number of patients receiving a fourth refill of their infliximab prescriptions because we compared utilization during the same calendar months for the 4 cohorts. Given that the decrease was transient, we surmise that it was caused by delays in refilling infliximab for the fourth time by patients in the policy cohort. The decrease could reflect an increase in the number of attempts to discontinue or taper treatment following remission, followed by re-initiation of treatment after a flare-up of symptoms (e.g., due to the nocebo effect). ${ }^{10,34}$ In the absence of clinical data, we are unable to confirm this explanation. Alternatively, this transient decrease might be a result of chance. Overall, we did not observe an increase in switching to a different biologic anti-inflammatory drug in January and February 2020, and the decline in infliximab refills was temporary; both are encouraging signs in terms of safety.

The increase in visits to specialists in rheumatology and dermatology for patients in the policy cohort was expected, because patients likely discussed switching with their specialists. Similar increases have been observed previously. ${ }^{10,14,35}$ While the use of conventional synthetic anti-inflammatory medications and NSAIDs was lower than in previous years, we consider this a positive signal; that is, fewer patients from the policy cohort experienced symptoms while receiving treatment with biosimilars.

Although the findings of our monitoring support the safety of the Biosimilars Initiative for infliximab users, further research is warranted to explore the potential longer-term impacts of a nonmedical switch to biosimilars. Indeed, the BC Ministry of Health has decided to extend the monitoring plan to a second year. In the absence of clinical data, we interpreted drug and health services utilization outcomes as proxies for patient health; more clinical research is needed to confirm our interpretation. Finally, this study focused on the use of infliximab in patients with inflammatory arthritis and psoriasis; monitoring switching policies for other medications and diseases is also important, as the effects in different populations may vary.

\section{Limitations}

This study had several limitations..$^{25}$ First, we did not test hypotheses and hence are unable to support or reject the null hypothesis of no difference in health services utilization after the policy was launched. As such, we did not adjust for patient characteristics, and differences in patient characteristics could lead to changes in clinical practice and health services utilization.

We used a normal probability model for our likelihood ratios and assumed that the cumulative incidences in the policy and historical cohorts were independent of each other. This assumption did not hold in our analysis because substantial numbers of patients were included in more than 1 cohort. We also took the average of the 3 likelihood ratios for comparisons 
with historical cohorts before comparing with the threshold value of 7.1. We used the threshold of 7.1 because in the case of a single likelihood ratio comparing 2 independent estimates, it corresponds to a $p$ value of 0.05 . The use of an average likelihood ratio for nonindependent estimates means that this correspondence does not hold and therefore the threshold of 7.1 that we used is likely more stringent than a threshold corresponding to a $p$ value of 0.05 when nonindependence and averaging are taken into account.

Another limitation is that we did not study the impact of the policy on individual patients; rather, we focused on its impact on the population level. In the case of the Biosimilars Initiative, focusing on populations (i.e., cohorts) allowed for signal detection that can be useful for planning further assessments of the policy. We also had no access to hospital data recorded during the last 2 months of follow-up for the policy cohort, which resulted in a shortened analytic period for this important outcome. In addition, follow-up was limited to 1 year after the initiative was launched and 6 months after all targeted patients were affected. This duration may be too short to detect delayed effects.

\section{Conclusion}

We observed no signal for negative impacts of phase 1 of the BC Biosimilars Initiative, a mandatory switch policy that targeted users of infliximab for inflammatory arthritis and psoriasis. This finding is encouraging, as it suggests that switching to the biosimilar infliximab has negligible or minimal effects on patient health. Our monitoring provided timely data from the first year of policy implementation. Detailed longer-term cohort studies, as well as studies planned for hypothesis testing, could provide additional assurance about the safety implications of the policy.

\section{References}

1. Prescribed drug spending in Canada, 2019: a focus on public drug programs. Ottawa: Canadian Institute for Health Information; 2019. Available: www.cihi.ca/en/ prescribed-drug-spending-in-canada-2019 (accessed 2020 Dec. 1)

2. Kobelt G, Kasteng F. Access to innovative treatments in rheumatoid arthritis in Europe: a report prepared for the European Federation of Pharmaceutical Industry Associations (EFPIA). Sweden: Lund University, European Federation of Pharmaceutical Industry Associations; 2009. Available: http://www.comparatorreports.se/ Access \%20to\%20RA\%20Treatments\%20October\%202009.pdf (accessed 2020 Dec. 1).

3. McCormick N, Wallace ZS, Sacks CA, et al. Decomposition analysis of spending and price trends for biologic antirheumatic drugs in Medicare and Medicaid. Arthritis Rheumatol 2020;72:234-41.

4. Mack A. Norway, biosimilars in different funding systems. What works? GaBi 7 2015;4:90-2.

5. Mulcahy AW, Hlavka JP, Case SR. Biosimilar cost savings in the United States: initial experience and future potential. Rand Health Q 2018;7:3.

6. Mansell K, Bhimji H, Eurich D, et al. Potential cost-savings from the use of the biosimilars filgrastim, infliximab and insulin glargine in Canada: a retrospective analysis. BMC Health Serv Res 2019;19:827-8.

7. Bae SC, Lee YH. Comparative efficacy and safety of biosimilar-infliximab and originator-infliximab in combination with methotrexate in patients with active rheumatoid arthritis: a meta-analysis of randomized controlled trials. Int 7 Rheum Dis 2018;21:922-9.

8. Sepriano A, Regel A, Van Der Heijde D, et al. Efficacy and safety of biological therapy and target synthetic DMARDs: a systematic literature review informing the 2016 update of the ASAS/EULAR recommendations for the management of axial spondyloarthritis. Artbritis Rheumatol 2016;68(Suppl 10):911-4.

9. Komaki Y, Yamada A, Komaki F, et al. Efficacy, safety and pharmacokinetics of biosimilars of anti-tumor necrosis factor-alpha agents in rheumatic diseases: a systematic review and meta-analysis. F Autoimmun 2017;79:4-16.
10. Convertino I, Lucenteforte E, Gini R, et al. Utilisation patterns and clinical impact of the introduction of infliximab-biosimilar in Tuscany, Italy: real world evidence following the recommendation of switching for non-medical reasons. Clin Exp Rheumatol 2021;39:753-62.

11. Glintborg B, Sorensen IJ, Loft AG, et al. A nationwide non-medical switch from originator infliximab to biosimilar CT-P13 in 802 patients with inflammatory arthritis: 1-year clinical outcomes from the DANBIO registry. Ann Rheum Dis 2017;76:1426-31.

12. Glintborg B, Sorensen J, Hetland ML. Does a mandatory non-medical switch from originator to biosimilar infliximab lead to increased use of outpatient healthcare resources? A register-based study in patients with inflammatory arthritis. RMD Open 2018;4:e000710.

13. Glintborg B, Loft AG, Omerovic E, et al. To switch or not to switch: results of a nationwide guideline of mandatory switching from originator to biosimilar etanercept. One-year treatment outcomes in 2061 patients with inflammatory arthritis from the DANBIO registry. Ann Rheum Dis 2019;78:192-200.

14. Glintborg B, Ibsen R, Bilbo REQ, et al. Does a mandatory non-medical switch from originator to biosimilar etanercept lead to increase in healthcare use and costs? A Danish register-based study of patients with inflammatory arthritis. RMD Open 2019;5:e01016.

15. Feagan BG, Lam G, Ma C, et al. Systematic review: efficacy and safety of switching patients between reference and biosimilar infliximab. Aliment Pharmacol Ther 2019;49:31-40.

16. Cohen HP, Blauvelt A, Rifkin RM, et al. Switching reference medicines to biosimilars: a systematic literature review of clinical outcomes. Drugs 2018;78:463-78.

17. Wiland P, Batko B, Brzosko M, et al. Biosimilar switching - current state of knowledge. Reumatologia 2018;56:234-42.

18. Halimi V, Daci A, Ancevska Netkovska K, et al. Clinical and regulatory concerns of biosimilars: a review of literature. Int 7 Environ Res Public Health 2020;17:5800.

19. Murdoch B, Caulfield T. The law and ethics of switching from biologic to biosimilar in Canada. 7 Can Assoc Gastroenterol 2020;3:228-33.

20. Numan S, Faccin F. Non-medical switching from originator tumor necrosis factor inhibitors to their biosimilars: systematic review of randomized controlled trials and real-world studies. Adv Ther 2018;35:1295-332.

21. Biosimilars Initiative for patients: current switch period. Victoria: British Columbia Ministry of Health. Available: https://www2.gov.bc.ca/gov/content/health/ health-drug-coverage/pharmacare-for-bc-residents/what-we-cover/biosimilars -initiative-patients (accessed 2022 Jan. 10).

22. How to submit a special authority request. Victoria: British Columbia Ministry of Health. Available: https://www2.gov.bc.ca/gov/content/health/practitioner -professional-resources/pharmacare/prescribers/special-authority/submitting-a -special-authority-request-information-for-prescribers (accessed 2020 Dec. 1).

23. Biosimilars Initiative: infliximab. Victoria: British Columbia Ministry of Health. Available: https://www2.gov.bc.ca/assets/gov/health/health-drug -coverage/pharmacare/patient-info-infliximab_final.pdf (accessed 2022 Jan. 10).

24. Dormuth CR, Fisher A, Carney G. A rapid monitoring plan following a shift in coverage from brand name to biosimilar drugs for rheumatoid arthritis in British Columbia. Pharmacoepidemiol Drug Saf 2020;29:796-802.

25. Fisher A, Kim JD, Dormuth CR. Rapid monitoring of health services utilization following a shift in coverage from brand name to biosimilar drugs in British Columbia — an interim report. Pharmacoepidemiol Drug Saf 2020;29:803-10.

26. Province declares state of emergency to support COVID-19 response [news release]. Victoria: BC Gov News/BC Public Safety and Solicitor General; 2020 Mar. 18. Available: https://news.gov.bc.ca/releases/2020PSSG0017-000511 (accessed 2020 Dec. 1)

27. Impact of COVID-19 on Canada's health care systems [homepage on Internet]. Ottawa: Canadian Institute for Health Information; 2021 Dec. 9. Available: https://www.cihi.ca/en/covid-19-resources/impact-of-covid-19-on-canadas -health-care-systems (accessed 2022 Jan. 1).

28. Al Zoubi S, Gharaibeh L, Jaber HM, et al. Household drug stockpiling and panic buying of drugs during the COVID-19 pandemic: a study from Jordan. Front Pharmacol 2021;12:813405.

29. Karlsson P, Nakitanda AO, Löfling L, et al. Patterns of prescription dispensation and over-the-counter medication sales in Sweden during the COVID-19 pandemic. PloS One 2021;16:e253944.

30. Elbeddini A, Hooda N, Yang L. Role of Canadian pharmacists in managing drug shortage concerns amid the COVID-19 pandemic. Can Pharm 7 2020; 153:198-203.

31. Morgan S, Yan L. Income-based drug coverage in British Columbia: the impact on private and public expenditures. Healthc Policy 2006;2:e129-e153. 
32. Valido A, Silva-Dinis J, Saavedra MJ, et al. AB1231 efficacy and cost analysis of a systematic switch from originator infliximab to biossimilar CT-Pp13 of all patients with inflammatory arthritis from a single centre. Ann Rheum Dis 2018;77(Suppl 2):1712-3

33. Goodman SN. Toward evidence-based medical statistics. 2: The Bayes factor. Ann Intern Med 1999;130:1005-13.

34. Fleischmann R, Jairath V, Mysler E, et al. Nonmedical switching from originators to biosimilars: Does the nocebo effect explain treatment failures and adverse events in rheumatology and gastroenterology? Rheumatol Ther 2020;7: $35-64$.

35. Phillips K, Juday T, Zhang Q, et al. Economic outcomes, treatment patterns, and adverse events and reactions for patients prescribed infliximab or CT-p13 in the Turkish population. Ann Rheum Dis 2017;76 (Suppl 2):835.

Affiliation: Department of Anesthesiology, Pharmacology and Therapeutics (Fisher, Kim, Dormuth), University of British Columbia, Vancouver, BC

Contributors: Anat Fisher contributed substantially to conception and design of the study and to acquisition, analysis and interpretation of data, and drafted the article. Jason Kim contributed substantially to the analysis and interpretation of data. Colin Dormuth contributed substantially to conception and design of the study and to acquisition, analysis and interpretation of data. All authors revised the article critically for important intellectual content, gave final approval of the version to be published and agreed to act as guarantors of the work.

Funding: This project was funded by the British Columbia Ministry of Health, Pharmaceutical, Laboratory \& Blood Services Division, as part of a plan for evaluating the impact of a change in coverage for biosimilar medications. All inferences, opinions and conclusions drawn in this article are those of the authors and do not reflect the opinions or policies of the British Columbia Ministry of Health.
Data sharing: The authors do not have permission to share data from this study. The data that support the findings of this study are available from Population Data BC (https://www.popdata.bc.ca/), but restrictions apply to the availability of these data, which were used under licence for the current study and so are not publicly available.

Content licence: This is an Open Access article distributed in accordance with the terms of the Creative Commons Attribution (CC BY-NC-ND 4.0) licence, which permits use, distribution and reproduction in any medium, provided that the original publication is properly cited, the use is noncommercial (i.e., research or educational use), and no modifications or adaptations are made. See: https://creativecommons.org/licenses/by-nc-nd/4.0/

Acknowledgements: The authors thank Tracey Hooper and Ellen Reynolds for presubmission editing assistance. The British Columbia Ministry of Health, facilitated by Population Data BC, approved access to and use of BC data for this study. All inferences, opinions and conclusions drawn in this manuscript are those of the authors, and do not reflect the opinions or policies of the data stewards. British Columbia data sources were as follows (https:// www2.gov.bc.ca/gov/content/health/conducting-health-research-evaluation/ data-access-health-data-central): 1 . British Columbia Ministry of Health [creator] (2020): Medical Services Plan (MSP) Payment Information File. BC Ministry of Health [publisher]. MOH (2019); 2. British Columbia Ministry of Health [creator] (2020): PharmaNet. BC Ministry of Health [publisher]. Data Stewardship Committee (2019); 3. Canadian Institute for Health Information [creator] (2020): National Ambulatory Care Reporting System. BC Ministry of Health [publisher]. MOH (2019); 4. Canadian Institute for Health Information [creator] (2020): Discharge Abstract Database (Hospital Separations). BC Ministry of Health [publisher]. MOH (2019); 5. British Columbia Ministry of Health [creator] (2020): Consolidation File (MSP Registration \& Premium Billing). BC Ministry of Health [publisher]. MOH (2019).

Supplemental information: For reviewer comments and the original submission of this manuscript, please see www.cmajopen.ca/content/10/1/ E109/suppl/DC1. 